\title{
Effect Analysis of Speed Guidance Control at Urban Expressway Intersection
}

\author{
Dashan Chen, Liguo Xia, Xiaoxian Yu and Chenglu Yang \\ School of Transportation Engineering, Huaiyin Institute of Technology, Jiangsu \\ Huai'an 223003, China \\ shyhits@126.com
}

\begin{abstract}
As the backbone of metropolitan transportation, urban expressway shares a large proportion of long distance traffic flow. With the motorization processing, traffic congestion is becoming more and more serious. Intersections are the key interrupted nodes of continuous traffic flow which are the direct inducement of freeway congestion. As an important method of active traffic management, speed guidance control was adopted to improve the road efficiency and safety level. Firstly, speed guidance mathematical model was established based on the traffic flow data and control strategy was also designed for the intersection. Then, online simulation system was built. Microscopic simulation platform was used to simulate the real world traffic. The macro dynamic traffic flow model was established and real-time data were exchange through the application programming interface among the three parts of the simulation system. Next, control effects were analyzed in detail. The results show that vehicle travel time and traffic delay greatly decreased. Emissions of pollutants also have been significantly reduced. Finally, this paper puts forward suitable proposals for the implementation of speed guidance control in China.
\end{abstract}

Keywords: traffic management; urban expressway; high speed intersection; speed guidance; microscopic simulation

\section{Introduction}

More than one million people died in the traffic accidents each year around the word, and much more were injured or disabled. Traffic participants, road facilities, weather conditions and unreasonable management have direct or indirect influence on traffic accidents [1]. Vehicle speed has been views as a key risk factor on road traffic injuries. Expressway system has become the urban mass rapid transit corridor and the main framework of urban road systems. Decreasing delay and improving throughput performance are great significance to improve the operation efficiency of expressway system. As the backbone of the city road network, urban expressway shares large proportion of the traffic. In Beijing, major urban expressway accounts for only $8 \%$ of the total length, but carries nearly $50 \%$ of the traffic flow; in Shanghai, only $5 \%$ bears more than $35 \%$ of the city traffic traveling [2]. Urban expressway plays a vital role in the urban road network which gradually shifted from the large-scale infrastructure construction to refinement traffic management. With the traffic demand rapid growing, much more congestion and traffic accidents, integrated active traffic management should be introduced in the background of coordination between road and vehicle [3,4]. As an important part of the active traffic management, the speed guidance control has certain positive significance to improve the expressway capacity, reduce the accident risk and decrease even eliminates traffic congestion.

With the developing of urban expressway, it is urgent to manage them scientifically and bring in some innovation technology. Based on the conducted traffic theory of control 
and safety, many scholars have made great contribution to the safe and efficient operation of expressway. When the speed guidance control involved in the traffic management system, traffic flow characteristics change a lot, some basic theory need to be further studied. Traditional variable speed limits control essentially provides a driving speed choice range for the driver [5-7]. During the range, the driver can arbitrarily decided vehicle speed. According to the active traffic management, more precise driving speed value had been settled, and the vehicle is guided which can reach the goal of safety and efficiency in the traffic system. Speed guidance control essentially provides the best driving speed value advice for the driver, so the traffic characteristic must be changed radically $[8,9]$. Traditional traffic flow model need to change. More large scale, scope and interactive relationship between vehicles change the driving behavior.

Geometric properties, traffic volume, vehicle speed and weather condition are the important factors influencing the occurrence of accidents. At present, most of the accident models parameters are calibrated by linear regression model which cannot fully reflect the accidents randomness and dispersion. Static accident data is used to establish the accident models which lack the prediction of short time accidents. It is necessary to predict the real-time road accidents by dynamic traffic flow. Through the study of accident risk factors, the probability of the accident could be reduced before the accidents really occur. With the rapid development of traffic information collection and processing technology, traffic demand, facility design, control measures and traffic participants are comprehensive considered in the experimental traffic engineering which analyses the effect of speed guidance control from the angle of vehicle microscopic driving behavior. Therefore, the microscopic traffic simulation has a natural advantage because its aims at the multi information observation of complicated traffic phenomena through the reproduction and the emergence analysis to reveal the speed guidance control.

According to traffic characteristics analysis, modeling traffic flow, and constructing the experimental platform, studies on intervention accident risk have been done in several aspects such as continuous data analysis, controlled experiments, behavioral modeling and control effect comparison to evaluate the results of expressway traffic flow evolution characteristics under speed guidance control.

\section{Literature Review}

Vehicle driving speed has been viewed as key risk factor on traffic injury. The speed not only has influence on the risk of traffic accidents also associated with the severity of accidents [10]. If the vehicle speed is higher than average speed the risk of accident is greater. Over speed or improper speed almost universally considered to be the main causes of traffic accidents. Through the Power model Nilsson reveals the relations between average speed and traffic accident. The results show that the average speed increased by $5 \%$ result in the number of accidents increased by about $10 \%$, and the number of deaths related accidents increased by about 20\% [11]. With the increase of vehicle speed, road traffic safety gradually reduces. However, Fildes and Lee think the cause of accident severity and cause of the accident should be distinguished carefully [12, 13]. They believe the real reason affecting the traffic safety is the discrete velocity of the vehicles. Pei research shows that dispersion of vehicle speed is one of the key important accident factors. Feng gets the same conclusion through the real-time traffic data, and he suggests that stability and safety can be improved by reducing vehicle speed discrete. Through the Bias classification and non parametric density function modeling, Oh finds that more accidents would happen if 5 minutes speed standard deviation is higher. The possibility of accidents could be reduced by decreasing the vehicle speed variance [14].

Accident model established by Golob considers average flow, flow changes, the median speed and speed changes; Kockelman builds the model adopting binomial regression method by analyzing the mean speed and speed change factors. Based on 
average occupancy and speed variance, model founded by Abdel-Aty can identify about $68 \%$ accidents [15]. At present, researchers mainly focus on accident modes, although some scholars have involved risk of accident that they did not notice the effects of traffic flow characteristics, driving behavior, and the capacity caused by dynamic speed control. Vehicle speed control has been put into operation in Germany, Holland and other developed countries. In addition to fixed speed control, variable speed limit control has been widely applied which can be suit for many kinds of roads, weather and the complicated traffic situation. Research on variable speed limit has always been a hot topic in the field of traffic control and management. Simulation methods such as kinetic model and cellular automaton model are used to reproduce the macroscopic traffic flow phenomena. Carlson and $\mathrm{Lu}$ et al conduct the research using stochastic model, traffic platoon model and improved traffic flow dynamics model on the variable speed limit control $[16,17]$. Meanwhile, improved METANET model is adopted by Papageorgiou to study control effect on traffic flow of variable speed limit from the application view [18].

It is difficult to precisely express microscopic traffic rules under speed guidance control because above methods have the following deficiencies such as too much theories assumptions, simple driving behavior modeling rules, and the adaptability of multigranular modeling. At present, most of the speed control research focus on variable speed limits which mainly studies the speed control measures in adverse weather conditions. In essence, new speed control methods could be introduced to guide the vehicle driving speed rather than restrict it. As one of the important methods of active traffic management, speed guidance caters to more precise traffic management needs.

\section{Speed Guidance Control at Intersection}

Through the reasonable speed guidance, the vehicle can choose the proper driving speed to pass through the intersection in green light time which can effectively reduce abnormal deceleration, cut down air pollution and improve the efficiency of the intersection.

Detectors are arranged at upstream of the intersection to collect the vehicle information. Speed guidance value is calculated based on the current signal light timing. When the vehicle comes in green light time, the speed needs no adjustment. If the vehicle is expected to arrive at the intersection in red light, speed guidance control could be used to accelerate or decrease the driving speed then the vehicle could get through the intersection without stop. Detectors are set near the intersection to obtain relevant information about the vehicles, and the message exchanges then the optimal driving speed is supplied to the drivers. Simulation model of the actual expressway is established based on the related theory and technology of mathematics, physics, and computer simulation. Traffic flow data were obtained through the survey and then VISSIM was employed to build the simulation model. Further, model calibration and verification were carried out carefully according to the traffic speed and volume.

Control-oriented macro dynamic traffic flow model describes the relationship among traffic flow over space and time even traffic control variables. LW model was proposed by British scholar Lighthill and Whitham in 1955. Against the defects, Payne proposed dynamic relationship between speed and density. The model was further extended considering off-ramp, on-ramp and lane change factors by Papageorgiou. Scholars between domestic and foreign have also proposed various models which are mostly around dynamic relationship between speed and density. Model proposed by Payne and Papageorgiou are widely used in practice. Second order dynamic METANET model is used to describe the traffic flow [19-22]. To make the calculated optimal speed guidance value more realistic and accurate, dynamic traffic flow model are described as Equation (1) and Equation (2): 


$$
\begin{aligned}
\rho_{i}(k+1) & =\rho_{i}(k)+\frac{T}{L_{i} \lambda_{i}}\left(\rho_{i-1}(k) v_{i-1}(k)-\rho_{i}(k) v_{i}(k)+r_{i}(k)-s_{i}(k)\right) \\
v_{i}(k+1) & =v_{i}(k)+\frac{T}{\tau}\left(u_{i}(k)-v_{i}(k)\right)+\frac{T}{L_{i}} v_{i}(k)\left(v_{i-1}(k)-v_{i}(k)\right) \\
& -\frac{1}{\tau}\left(\frac{v T}{L_{i}} \frac{\rho_{i+1}(k)-\rho_{i}(k)}{\rho_{i}(k)+\kappa}\right)
\end{aligned}
$$

Where $i$ is road segment index, $k$ is time interval index, $T$ is the time step used for data collection, $L_{i}$ is length of road segment. $\tau, v, \kappa$ are the model parameters, $\tau$ is a time constant, $v$ is the anticipation constant and $\kappa$ is model parameters which are equal at a segment. $\rho_{i}(k)$ is the traffic density of road segment $i$ at time index $k, v_{i}(k)$ is the mean speed of road segment $i$ at time index $k, r_{i}(k)$ is the metering flow rate of road segment $i$ at time index $k, s_{i}(k)$ is the total off-ramp flow rate of road segment $i$ at time index $k, u_{i}(k)$ is the desired control speed of road segment $i$ at time index $k$ and $\lambda_{i}$ is the number of lanes of road segments.

The paper determines the origins flow and upstream speed parameters by the ramp length and queue of upstream cell segment. The models are listed as Equation (3) to Equation (5):

$$
\begin{array}{r}
w_{0}(k+1)=w_{0}(k)+T\left(d_{0}(k)-q_{0}(k)\right) \\
q_{0}(k)=\min \left\lceil d_{0}(k)+w_{0}(k) / T, Q_{0} \frac{\rho_{\max }-\rho_{\mu, 1}(k)}{\left.\rho_{\max }-\rho_{c r i t, \mu}\right]}\right. \\
v=\min \left[(1+\alpha) v_{c r i t, m}(k), v_{\text {free }} \exp \left[-\frac{1}{\alpha_{m}}\left(\frac{\rho_{\mu, 1}(k)}{\rho_{c r i t, u}}\right)^{\alpha_{m}}\right] \mid\right.
\end{array}
$$

Where $Q_{0}$ is the onramp flow capacity, $\rho_{\max }$ is the maximum density of onramp, $\rho_{\text {crit }, u}$ is the critical density of onramp at which the traffic flow becomes unstable, $\rho_{u, 1}(k)$ is the density of mainline which segment the onramp linked, $v_{c r i t, m}$ is the critical speed of mainline at which the traffic flow becomes unstable, $v_{\text {free }}$ is the speed of freely traffic flow, $w_{0}(k)$ is the maximum number of vehicles stored in onramp and $d_{0}(k)$ is the onramp demand flow.

In the speed guidance control online simulation system, VISSIM software is used to simulate the real world traffic. The macro dynamic traffic flow model is established in MATLAB software. Data and control strategies were exchange through the API interface among VB.NET, VISSIM and MATLAB. According to the functional orientation, online simulation system was divided into four modules: simulation module, strategy module, interface module and database module. The structures of the simulation system as well as the relationship among the modules were shown in Figure 1. 


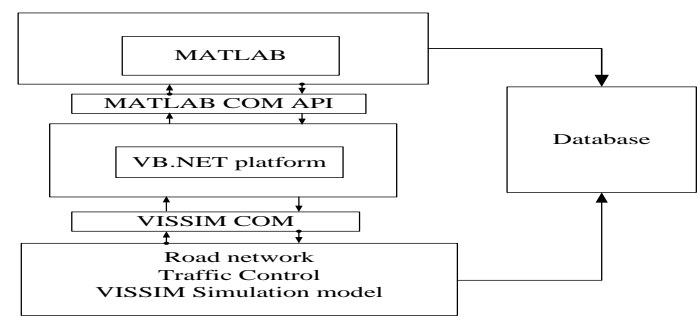

\section{Figure 1. Framework of Online Simulation System}

When the vehicle pass the detector, message of signal light, vehicle speed and cycle time are acquire to judge whether the vehicle can pass the intersection. If the vehicle cannot once get through the intersection, speed guidance control will intervene. Control schemes are shown in equation (6) to (9).

$$
\begin{gathered}
t_{1}=\frac{L}{v_{0}} \\
t=t_{0}+t_{1} \\
T=t_{1}-\left[\frac{t_{1}}{c}\right] c \\
v_{c}=\frac{L}{t_{1}}
\end{gathered}
$$

Where $L$ is the distance between the detector and intersection, $t_{0}$ is the vehicle arrive time, $C$ is the signal light cycle, $v_{0}$ is the Initial vehicle speed. $c_{1}$ is the length of signal green time, $c_{2}$ is the length of signal red and yellow time. $v_{c}$ is the value of speed guidance control, the minimum and maximum values are $20 \mathrm{~km} / \mathrm{h}, 80 \mathrm{~km} / \mathrm{h}$ respectively. The $T$ value is in the range of $\left[0, c_{1}\right]$, vehicles do not need the speed guidance control, and they can pass through the intersection directly. If the $T$ drops in $\left[c_{1}, c_{2}\right]$, speed guidance is applied to control the vehicle speed to ensure that the vehicles get through the intersection without any stop.

Traffic data was acquired to evaluate the road traffic efficiency and safety level, Vehicle Specific Power (VSP) had been adopted to evaluate the road environmental effect under speed guidance control. VSP refers to the engine quality power output which was proposed by Jimenez-Palacios in 1999 [39]. Variation of the kinetic energy, potential energy changes, rolling friction and air resistance are taking into account to establish the value of engine work. Instantaneous driving condition and vehicle emission had been linked closely then it is possible to compare and analyze the emissions data. The calculation formula listed as follows:

$$
V S P=v\left[a\left(1+\varepsilon_{i}\right)+g(\text { grade })+g C_{R}\right]+\frac{1}{2} \rho_{a} \frac{C_{D} A}{m}\left(v+v_{w}\right)^{2} v
$$

Where $\mathrm{m}$ is the vehicle weight, $\mathrm{V}$ is the vehicle speed, $v_{w}$ is the wind speed, $C_{D}$ is the drag coefficient, A is the cross sectional area, $\rho_{a}$ is the air density, $\varepsilon_{i}$ is the rolling quality coefficient, $\mathrm{h}$ is the vehicle altitude, $\mathrm{g}$ is the acceleration of gravity, $C_{R}$ is the roll damping coefficient and $\mathrm{a}$ is the vehicle acceleration. 
VSP is a directional quantity, when the vehicle was braking; the power demand of VSP is negative because its force direction is in the opposite direction. Similarly, the power demand of VSP characterization is positive when the vehicle speed is accelerating. Based on the real time data, velocity and acceleration, effect on environment under speed guidance of expressway had been evaluated indirectly.

\section{Simulation Analysis and Results}

Microscopic traffic simulation software VISSIM developed by the PTV company is adopted to simulate traffic operation. VISSIM uses 2D or 3D animation to show the running condition of vehicles. Based on time driven and driving behavior modeling, VISSIM can be through variety of traffic parameters such as lane setting, traffic structure, traffic signal setting, traffic signal timing, transit site distribution, and the relationship between the traffic conditions to build the virtual traffic world. Travel time, delay and emissions are used to evaluate the traffic engineering facility design and traffic control scheme.

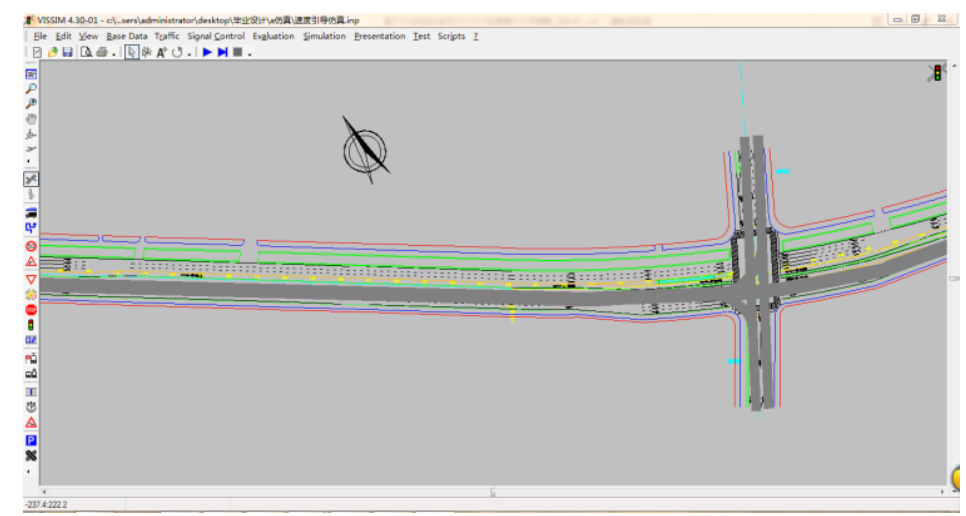

Figure 2. Traffic Simulation Model

Xiangyu expressway is used as the research and evaluation object under speed guidance control. Layout of expressway, signal phase, classification of vehicle types and traffic volume has been investigated before establishing the simulation model. After Xiangyu expressway road network had been drawn by AutoCAD, the base map is imported into VISSIM to establish the traffic simulation model as shown in Figure 2.

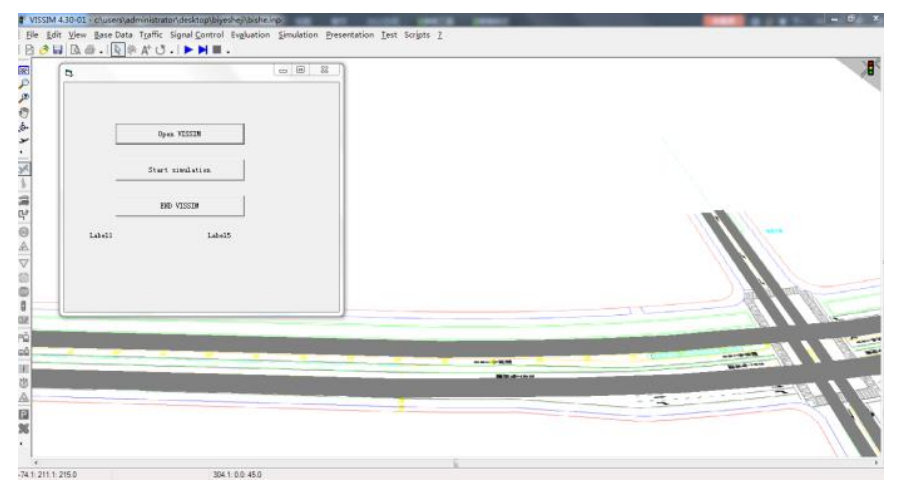

\section{Figure 3. Analysis Platform of Speed Guidance Control}

During the simulation process, travel time, delay time, average queue length and the maximum queue length decreased obviously when the expressway was under speed guidance control. The average travel time decreased more than $23 \%$ when the vehicle 
were controlled in the expressway as shown in Figure 4, so the speed guidance can save a lot of travel time.

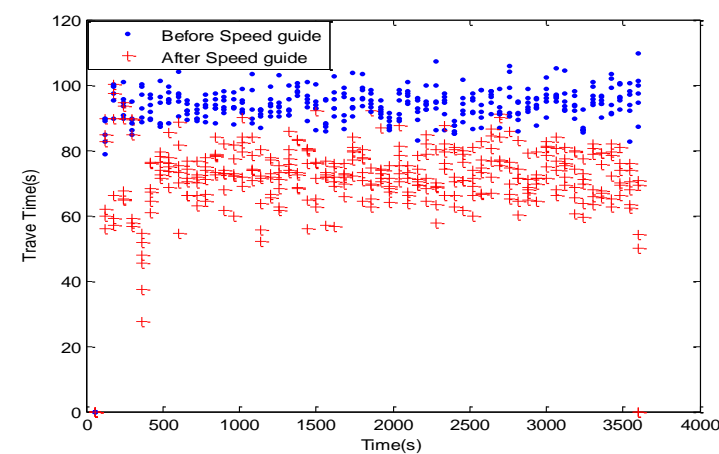

Figure 4. Vehicle Parking Time and VSP Value Change

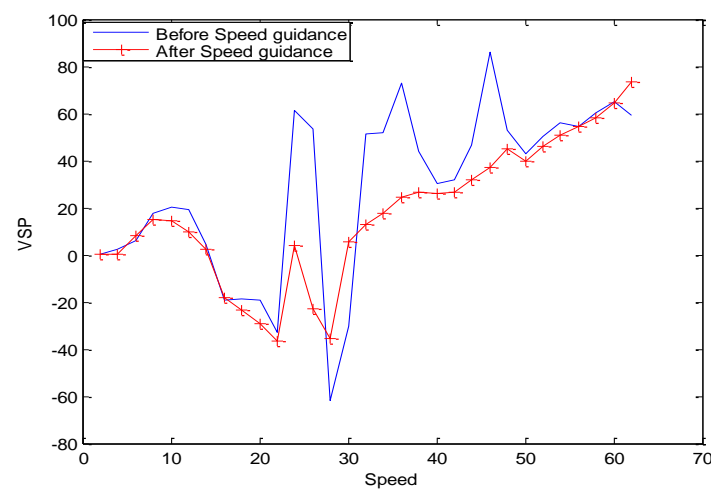

Figure 5. VSP Value Change

In order to reflect the efficiency of speed guide control, control delay is selected as the evaluation index. As shown in Figure 6, vehicle delay distribution changed significantly, the average vehicle delay optimization proportion is more than $30 \%$.

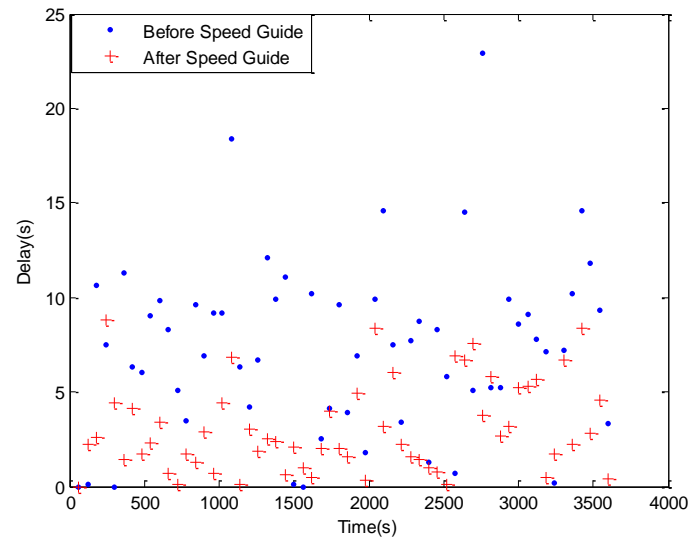

Figure 6. Traffic Delay before and after Speed Guidance Control

Parking time is an effective evaluation index about traffic efficiency. Through the speed guidance, the average parking time is obviously shortened as shown in Figure 7. 


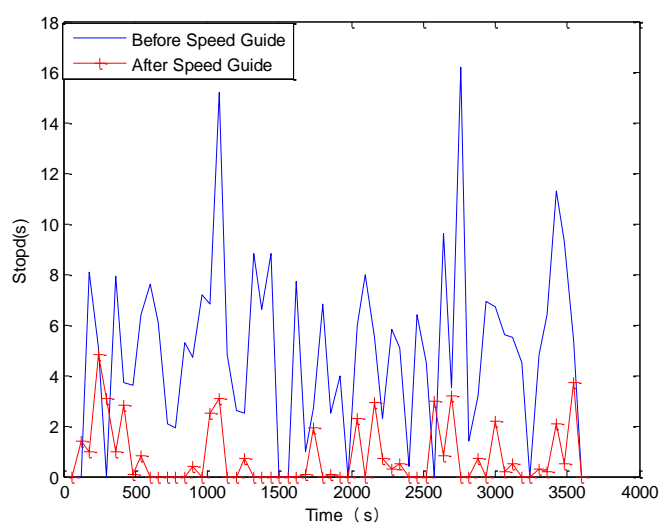

Figure 7. Comparison of Parking Delay before and after Speed Guidance

\section{Conclusion}

By analyzing the traditional variable speed limits, speed guidance control have been proposed. Vehicles generally choose the driving speed among a range under the speed limits. In contrast, speed guidance control plan to provide a unique speed value for the drivers that is quite different from the other methods. Expressway intersection has been adopted as the research object. Firstly, traffic flow data has been collected which could be used to calibrate the macro dynamic traffic flow model. The original data should be cleaned carefully to improve data quality. Secondly, control algorithm is designed then the vehicles could adjust the speed to maximize reducing parking time in the intersection without speed change greatly. Finally, traffic model is established in vissim and the model is used to simulate the real traffic flow. Data exchange platform is also built to test the effects of speed guidance control.

Based on the traffic information of the downstream intersection, the driver can adjust the speed in advance and vehicles pass through the intersection much more smoothly under speed guidance control. The simulation results show that the road traffic safety and efficiency are improved, and the emission of harmful gas is also reduced.

However, the following aspects need to do further research. First of all, traffic flow has a great influence on control effect. When the traffic volume is too large, speed guidance may play a negative effect. So sensitivity analysis of traffic volume on the simulation results is one of the most important research contents in the further. Obedience of the driver to the speed guidance value directly affects the effect, the rate of drivers obey the information should be also considered in reality. Second, the speed guidance mainly serves the normal traffic flow, how to adjust the control strategies when accidents occur is another research content. At last, the research carried out based on microscopic simulation model which affected by the results of the calibration and validation on the traffic model. The results of speed guidance control have certain subjectivity. How to apply speed guidance control into practice are problems to be solved in the next step.

\section{Acknowledgements}

This research is supported by the National Natural Science Foundation of China (Project No. 51408252). The authors would like to express their sincere thanks to the anonymous reviewers for their helpful comments and valuable suggestions on the paper. 


\section{References}

[1] C. Fuhs, "Synthesis of Active Traffic Management Experiences in Europe and the United States", (2010) Washington, D. C. Federal Highway Administration.

[2] D. H. Wang, X. W. Chen and S. H. Yang. "Analysis of Traffic Bottleneck on Beijing Urban Expressway", Proceedings of 7th International Conference of Chinese Transportation Professionals, (2007) August 2-5, Beijing.

[3] A. Hegyi, D. Schutter and H. Hellendoorn, "Optimal Coordination of Variable Speed Limits to Suppress Shock Waves", Transportation Research Record, vol. 1852, no.1, (2004), pp.167-274.

[4] A. Hegyi, D. Schutter and H. Hellendoorn, "Model Predictive Control for Optimal Coordination of Ramp Metering and Variable Speed Limits", Transportation Research Part C, vol. 13, (2005), pp.185209.

[5] H. C. Gan, L. J. Sun and Y. Hao, "A Case Study of Urban Expressway Traffic Flow Based on Hig-order Continuum Model”, Journal of Tongji University Natural Science, vol. 35, no.5, (2007), pp. 602-606.

[6] M. Hadiuzzaman and T. Z. Qiu, "Cell Transmission Model based Variable Speed Limit Control for Freeways", Journal of Civil Engineering, vol. 40, no.1, (2013), pp. 46-56.

[7] G. Bel and J. Rosell. "Effects of the $80 \mathrm{~km} / \mathrm{h}$ and Variable Speed Limits on Air Pollution in the Metropolitan Area of Barcelona", Transportation Research Part D: Transport and Environment, vol. 23, no. 1 , (2013), pp. 90-97.

[8] M. Hadiuzzaman, T. Z. Qiu and X. Y. Lu, "Variable Speed Limit Control Design for Relieving Congestion Caused by Active Bottlenecks", Journal of Transportation Engineering, vol. 139, no. 4, (2012), pp. 358-370.

[9] B. G. Heydecker and J. D. Addison, "Analysis and Modelling of Traffic Flow under Variable Speed Limits”, Transportation Research Part C: Emerging Technologies, vol. 19, no. 2, (2011), pp. 206-217.

[10] J. Buddemeyer, R. K. Young and B. Dorsey-Spitz, "Rural Variable Speed Limit System for Southeast Wyoming”, Transportation Research Record, vol. 2189, no. 1, (2010), pp. 37-44.

[11] R. L. Bertini, S. Boice and K. Bogenberger, "Dynamics of variable Speed Limit System Surrounding a Bottleneck on a German Autobahn", Transportation Research Record, vol. 1978, no. 1, (2006), pp. 149159.

[12] C. Lee, B. Hellinga and F. Saccomanno, "Evaluation of Variable Speed Limits to Improve Traffic Safety”, Transportation Research Part C Emerging Technologies, vol. 14, no. 3, (2006), pp. 213-228.

[13] C. Lee and M. Abdel-Aty, "Testing Effects of Warning Messages and Variable Speed Limits on Driver Behavior Using Driving Simulator", Transportation Research Record, vol. 2069, no.1, (2008), pp. 55-64.

[14] N. J. Fudala and M. D. Fontaine, "Interaction between System Design and Operations of Variable Speed Limit Systems in Work Zones", Transportation Research Record, vol. 2169, no.1, (2010), pp. 1-10.

[15] M. Abdel-Aty, R. J. Cunningham and V. Gayah, "Dynamic Variable Speed Limit Strategies for Realtime Crash Risk Reduction on Freeways", Transportation Research Record, vol. 2078, no.1, (2008), pp. $108-116$

[16] R. C. Carlson, I. Papamichail and M. Papageorgiou, "Optimal Motorway Traffic Flow Control Involving Variable Speed Limits and Ramp Metering”, Transportation Science, vol. 44, no. 2, (2010), pp. 238-253.

[17] R. C. Carlson, I. Papamichail and M. Papageorgiou, "Local Feedback-based Mainstream Traffic Flow Control on Motorways Using Variable Speed Limits", IEEE Transactions on Intelligent Transportation Systems, vol. 12, no.4, (2012), pp. 1261-1276.

[18] M. Papageorgiou and I. Papamichail, "Overview of Traffic Signal Operation Policies for Ramp Metering”, Transportation Research Record, vol. 2047, no.1, (2008), pp. 28-36.

[19] M. J. Lighthill, and G. B.Whitham, "On Kinematic Waves II : A Traffic Flow Theory on Long Crowded Roads", Proceedings of the Royal Society of London, vol. 229, (1955), pp. 317-345.

[20] H. J. Payne, "Models of Freeway Traffic and Control", Simulation Council Proceedings, vol. 1, no.1, (1971), pp. 51-61.

[21] M. Papageorgiou, "Macroscopic Modeling of Traffic Flow on the Boulevard Paripherique in Paris", Transportation Research Part B, vol. 23, no.1, (1989), pp. 29-34.

[22] M. Papageorgiou and J. M.Blosseville, "Modeling and Real-Time Control of Traffic Flow on the Southern Part of Boulevard Peripherique in Paris", Transportation Research Part A, vol. 24, no.5, (1990), pp. 345-359. 


\section{Author}

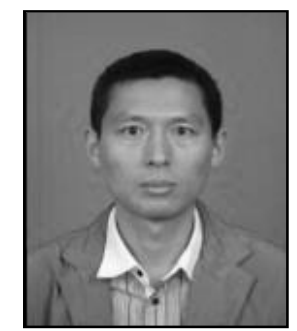

Dashan Chen, he works at Huaiyin Institute of Technology, was born in October 1983, received the B.S. and M.S. degrees from Chang'an University. He received the Ph.D. degrees from Tongji University, Shanghai, China, in 2012. He is an associate professor in the school transportation engineering. His research interests include traffic safety and intelligent transportation systems. 